\title{
Implementation of a care coordination system for chronic diseases
}

\section{Jung Jeung Lee, Sang Geun Bae}

Department of Preventive Medicine \& Public Health, Keimyung University School of Medicine, Daegu, Korea

Received: November 1, 2018

Revised: December 8, 2018

Accepted: December 18, 2018

Corresponding author:

Sang Geun Bae

Department of Preventive

Medicine \& Public Health,

Keimyung University School of

Medicine, 1095, Dalgubeol-daero,

Dalseo-gu, Daegu 42601, Korea

Tel: $+82-53-580-3894$

Fax: +82-53-580-3799

E-mail: baesg@dsmc.or.kr
The number of people with chronic diseases has been increasing steadily but the indicators for the management of chronic diseases have not improved significantly. To improve the existing chronic disease management system, a new policy will be introduced, which includes the establishment of care plans for hypertension and diabetes patients by primary care physicians and the provision of care coordination services based on these plans. Care coordination refers to a series of activities to assist patients and their families and it has been known to be effective in reducing medical costs and avoiding the unnecessary use of the hospital system by individuals. To offer well-coordinated and high-quality care services, it is necessary to develop a service quality assurance plan, track and manage patients, provide patient support, agree on patient referral and transition, and develop an effective information system. Local governance should be established for chronic disease management, and long-term plans and continuous quality improvement are necessary.

Keywords: Chronic disease; Patient care management; Referral and consultation; Transitional care

\section{Introduction}

Population aging has led to a steady increase in the number of people with chronic diseases, and the socio-economic costs due to the related complications, disabilities, and premature deaths, as well as direct medical costs, are rising rapidly [1]. Despite this current status, the management of chronic diseases has not improved significantly. For example, the blood pressure control rates of patients with hypertension and the glucose control rates of those with diabetes have remained at approximately $60 \%$ and $20 \%$, respectively [2]. To improve the above described problems in the management of chronic diseases, numerous public projects have been implemented in some regions. Although these projects have resulted in some achievements related to developing awareness on diseases and medication adherence $[3,4]$, this approach comprising sporadically implemented individual projects has not shown population-level effects in the South
Korean environment, where there are no incentives for healthcare providers to engage in chronic disease management [5].

Recently, the Ministry of Health and Welfare announced the plan for the "Pilot Project for Primary Care Chronic Disease Management," and it will introduce a system for the stepwise strengthening of chronic disease management based on primary care services. The current pilot project includes the establishment of care plans for patients with hypertension and diabetes by primary care physicians and the provision of care coordination services based on these plans. Care coordination refers to a series of activities that assist patients and their families in self-managing their health conditions and related psychosocial problems more effectively, coordinating their care among multiple health and community providers, bridging gaps in care, and receiving the appropriate level of care. Numerous countries, such as the United Kingdom (UK), the Netherlands, the United States, and Japan, have already introduced care coordination 
systems for the management of chronic diseases, or they have implemented care coordination services within the national healthcare system. Such measures have proven effective in reducing medical costs and avoiding the unnecessary use of the hospital system by individuals [6].

However, it is difficult to apply these foreign systems directly to South Korea. The reasons for the difficulty in introducing a care coordination system could include the nature of medical practice, which is based on a private-dominated supply system; opposition from the medical field against the introduction of the prospective payment system; and lack of experience and resources on chronic disease management. Therefore, it is important to introduce a system by adapting it to our conditions and circumstances. Nevertheless, because the conditions described above have been formed during the historical development of the South Korean healthcare system, these conditions should be considered in terms of overall chronic disease management, including care coordination.

This study aimed to examine the factors of care coordination in the management of patients with chronic diseases, and further investigate the conditions and plans for implementing the policy successfully in South Korea.

\section{Key elements of care coordination}

The Chronic Care Model (CCM) has been used widely as a guideline for effective chronic disease management since its emergence in the 1990s $[7,8]$. It emphasizes the need for change in the existing health and medical system for dealing with chronic diseases management, presenting the factors that are important appropriate chronic diseases management, such as linkage with community resources, reflecting on the characteristics of healthcare institutions, self-management support, design of a delivery system, decision-making support, and establishment of a clinical information system. This approach purports that the community and healthcare system should collaborate to improve treatment outcomes by creating "informed and activated patients" and "prepared and proactive practice teams," and developing a productive relationship between patients and the practice team. Studies have revealed that interventions based on the CCM have led to improvements in treatment outcomes changing the manner in which medical and care services are provided [7]. Therefore, the present study utilized the CCM to examine the elements necessary for care coordination and further discuss practical measures for South Korea.

\section{Structure and responsibilities of care coordination team}

The CCM model emphasizes that chronic diseases management should be organized, coordinated, and provided by the management team. Further, it is recommended to employ a population-based approach to influence treatment outcomes [9]. Such an approach should include a care plan based on complex and comprehensive assessments and rationales on the problems of patients, and it should involve cooperating with patients to identify hurdles, solve their own problems, and achieve service goals. Previous studies reported that the care coordination team is effective in the areas of heart failure, diabetes, treatment of the frail elderly, and mental health services integrated with chronic pain [10-14], further revealing that other healthcare professionals and office workers can play a partial role equivalent with that of physicians, in treating chronic diseases $[15,16]$.

The care coordination team could include the patient and his/ her family, the primary care provider, the care coordinator, and the assistant clinician, if necessary. These team members should fulfill their respective treatment responsibilities and further share responsibilities pertaining to intervention and follow-up observation. The first step in chronic disease management is to create a team that provides comprehensive care based on the needs of patients with a sense of responsibility.

However, it is difficult to construct and operate a team that provides care with a comprehensive responsibility for disease management in the current private sector-led healthcare delivery system in South Korea. Although the National Health Insurance Service is a single insurance provider in South Korea, the public sector's role in financing the healthcare system is low. Further, more than $90 \%$ of the beds for patients with acute diseases are owned by the private sector. In South Korea, neither can the government be responsible for all services, as in the case of National Health Service in UK, nor can each insurer assume the responsibilities, as in the United States, the Netherlands, and Japan. Although the labor cost for care coordinators could be financially aided by the National Health Insurance (NHI), the care services need to be coordinated through a network of service providers in each region, and individual institutions responsible for the coordination of care services should modify them based on the local situation.

Currently, among the various institutions that could shoulder the responsibility of coordinating care services, possible candidates could be public organizations, such as public health centers and the NHI, and civil organizations, including local medical associations. Public health centers could be 
advantageous for providing comprehensive services because they can be linked easily with community resources. Associations of service providers, such as local medical associations, could be responsible for service quality assurance in clinical terms, because their accessibility could be used to foster cooperation between service providers. Although the NHI can stably employ care coordinators, it is difficult to connect them with local communities. More importantly, there is no consensus on whether care coordinators employed by the NHI could be dispatched owing to the long-lasting conflict with service providers on medical payments. Thus, it is difficult to implement a care coordination system without the cooperation of or at least agreement by providers [17].

A more important issue than that pertaining to the care coordination team is the need to establish local governance based on the involvement of multiple service providers to provide and coordinate comprehensive services from the viewpoint of patients. It is difficult for one institution to have comprehensive responsibility for patients in the South Korean context. Therefore, it is necessary for one institution to lead the coordination of care services and simultaneously encourage the participation of other service providers. Furthermore, the participation of community health workers should be guaranteed to provide comprehensive services and to communicate with service providers [17].

\section{Plan for service quality assurance}

In many primary healthcare institutions, care is not coordinated by merely enforcing a system and supporting the manpower and finances. The improvement of the coordination system requires concerted efforts. It is necessary to relocate the personnel and train new roles for care coordination, and further establish networks with numerous service providers and organizations in the local community. Moreover, the timely sharing of information between patients and various service providers should be ensured. These efforts would lead primary care providers to make decisions that improve care coordination.

The next step after establishing a care coordination team is to develop quality management plans and monitor the operations [18]. Planning should begin with clear goals such as receiving a reply on a consultation report each time after specialist referral, or contact with a patient within 3 days after hospital discharge. The quality indicators of chronic disease management projects that have been implemented thus far have directly measured changes in patients, including self-recognition of blood pressure or blood glucose levels, drug intake, health behaviors relating to nutrition and physical activities, and blood pressure and blood glucose control. On the other hand, process indicators that can directly show the status of care coordination, such as patient referral, report reply, and patient information sharing, have not been measured. Though not in the community, several cases of improvement through inter-department cooperation within regional cardiovascular centers have been reported by monitoring several indicators, including the time from hospital arrival to treatment for emergency patients with conditions such as myocardial infarction and stroke [19]. In the medical field, if consensus is reached between participating experts, quality indicators that reflect the clinical viewpoint could be identified within a short period.

The most important components of quality assurance for services over the long term is the content of the services offered by the care coordinator and the training of the workforce. However, it is unrealistic to conduct the educational programs developed in foreign countries in the absence of a care coordination service in South Korea. Indeed, it is logical to manage quality and educate personnel based on services that can be performed effectively owing to the implementation of the system. Several aspects of healthcare services need to be provided by experts, which requires vertical quality control. However, the care coordinator also needs to reflect on the needs of patients and to ensure access to services. The strict assessment and monitoring of the contents of the services offered is therefore essential. Simultaneously, it is important to provide autonomy to service provides to enable them to develop services to suit the needs of patients. Therefore, educational programs for personnel should be developed continually through linking theoretical education and practice of the services provided onsite, rather than through standardized processes. Thus, educational institutions for healthcare personnel should be able to cater to a sizeable number of students to meet the needs of onsite service providers. Considering that the size of the city and county is too small, and it would be appropriate to divide them into several districts.

\section{Tracking and management of patients}

The primary goals of care coordination are to manage the quality of care services provided at the time of patient referral and transition. Additionally it should enable service providers and related organizations, as well as patients, to have the information and resources they need for providing appropriate care. These tasks the core tasks of the care coordination team. The primary care provider can refer the patient to a specialist or other provider 
for services that he/she needs. Therefore, the primary care provider becomes primarily responsible for patient referrals and care coordination. On the other hand, with reference to interhospital transitions of patients, the primary service provider at the institution from which the patient is discharged, or the institution itself, is responsible for the transition. However, the direction of transition could be reversed, such as transition from a primary care facility to a hospital. When various service providers are involved in the treatment of patients, they are solely responsible for the individual services they provide, while it is often unclear who is responsible for coordinating the various services provided to the patient. Indeed, the care coordination team should assume this responsibility [18].

Care coordination requires the consideration of patient activities outside the institution to which the provider belongs. Thus, the recognition of these activities is essential in care coordination. The tracking of extra-organizational activities by patients begins with recording basic information on the referral and transition documentation. This task develops into strategies to evaluate and record the implementation of major steps, including scheduling consultation with a specialist, delivery of information for consultation, implementation of the referred consultation, and handing over a report after the referral and transition [18].

Because incidents occur suddenly, such as hospitalization and emergency room visits, it is difficult to acquire the necessary information in a timely manner if no information delivery system is established in advance. Patients could lack information on their illness or the doctor in charge of the treatment may not be available when the patients visit the hospital suddenly. An electronic record system can help in such cases, and a card that patients can carry in his/her wallet could provide all the important information to the medical staff. Furthermore, when the care coordinator intends to acquire the information on the entry and exit of patients, the care coordinator could rely on the hospital physician and the emergency room physician. However, it is the best to have a daily entry/discharge report from the hospital [18]. In the case of infected patients, an infection manager in the hospital can easily access and manage the information on such patients. However, currently, hospitals do not have a system for managing the information on the entry and discharge of patients with chronic diseases. Although the insurance claim data could be helpful, the acquired information would not be timely. Therefore, it is necessary to establish a system for tracking and managing patients in cooperation with local hospitals.

\section{Patient support}

Care coordination can be divided into multiple steps, such as logistic support in service delivery, clinical monitoring, and support for self-management or drug use. Particularly, case management and care coordination should be distinguished clearly. Nurses and other care managers in primary care clinics have been provided clinical support regarding clinical evaluation, follow-up observation, self-management support, and medication management. Case management primarily provides some care coordination functions for high-risk patients, while the care coordinator strives to complete patient referrals by handling logistic or financial hurdles. Moreover, the care coordinator allows patients to receive timely referred treatment, and further resolves problems faced by patients by delivering clinical information and tracking the referral process. Most benefits from patient support arise mainly from handling logistic steps or information on patient support related to patient referral or transition [18].

Numerous diseases, such as diabetes, require continuous and comprehensive management. The more severe the patient's condition is, the more likely he/she is to require services from other specialists and health and welfare services in addition to those offered in primary care clinics [20]. It is therefore important to develop linkages with community resources to meet the comprehensive needs of patients with chronic diseases, and to ensure the continuity and completeness of treatment [17]. Although the introduction of a care coordination system would help improve the community linkage, the first challenge in achieving the same is the conflict between the public health agencies and the private hospitals and clinics. Private clinics are commonly annoyed by patients' use of services at public health centers. However, it is difficult to link clinics with the community if they do not accept such practices. Indeed, minimal acceptance is essential [17].

The easiest way to strengthen the linkage of service providers to a local community is to conduct conversations on common issues. Private clinics commonly lack the information on services provided by public health centers. This is also observed between departments within a public health center. Therefore, it is necessary for individual institutions or organizations to share information on both private and public services provided in the local community, and to conduct subsequent meetings on case management. Currently, some meetings on case management are being conducted occasionally in public health clinics and public health centers, particularly regarding the visiting healthcare services project. In this case, the challenge lies in the participation 
of doctors. Even within the same institution, the participation of healthcare providers is insufficient. Although physicians could participate by creating a care plan for patients, the provision of the information related to the care, the mechanism to participate in the decision-making process, and the provision of incentives, if necessary, should be considered to improve the linkage and cooperation among healthcare providers.

\section{Agreement on patient referral and transition}

It is important for service providers to agree on the purpose and importance of referral, and their respective roles in patient referral and transition. The agreement begins by focusing on the service providers and institutions that are frequently referred to, and further building relationships with the major service providers in the community. Continuing conversations could lead to consensus to improve the referral system [21]. Further, relationships need to be established with providers of services such as social services, health behavior support, and peer support, as well as with major specialists, hospitals, and emergency medical institutions [18]. The inclusion of some of the service providers from the community in the care coordinator team could help facilitate communication between providers [17].

Even if service providers agree with the standardized format and method of patient referral or transition, the practice needs to be managed continuously. If a hospital or clinic is open or closed and new public services are created, the newcomers should be asked to agree on the existing methods or new methods need to be agreed upon. Although the agreed expectations could be formalized in writing, dialogue between the parties as well as the individual relationships formed accordingly are crucial factors. Official regulations or guidelines are important, but it is more important to build a network of service providers. Although guidelines agreed in writing are important, there could be some unsettled issues, and informal consent about the details is also important. In this case, human relationships play an important role. The relationship between providers has weakened in comparison to that in the past, which has become an obstacle to service linkage among providers [22]. The same is applicable to South Korea. Another manner in which agreed expectations can be systemized is the use of an electronic referral system [23], especially if appropriate consent is obtained, unnecessary referrals are reduced, duplicate surveys are avoided, and optimal treatment is provided after referral and discharge $[24,25]$.

\section{Information system}

A crucial factor in successful patient referrals and transition is to have the information that service providers require for providing their services. Hospitals, clinics, and public health centers used different electronic medical record systems, and it is difficult to standardize medical information, which in turn is a hurdle in establishing an electronic information system [23]. If the practice team is organized and consensus is established among service providers, information that is essential for the referral and transition of patients could be standardized [24]. The electronic referral system can help ensure that this information is delivered in a timely manner, incorporating the agreed guidelines for the referral and transition of patients to reduce unnecessary transfer and transitions of patients. Furthermore, it could be a trusted source of information for primary care providers and specialists [23]. However, the delivery of this information could be performed through a pen-and-paper-based medium by structuring and standardizing referral requests and medical notes.

Although the amount and accessibility of the delivered information is important, ongoing management of the information system is more vital. In addition to the organizations participating at the beginning, as local networks expand, more providers could participate. Different regions have different conditions and additional needs may be added over time. Accordingly, to increase the usability of the information system, it is necessary to be able to respond immediately to such changing conditions. It is necessary to set up a system which allows various stakeholders to access the information system and develop various applications (apps). With the recent increase in the use of cloud services or open-source programs, computer nonspecialists are developing their own apps. If health professionals can participate directly in app development, they will be able to respond to a variety of needs and changes in the field.

Service providers require the information system to obtain any information they need. Moreover, patients should have access to the information system to enable them to provide information on their diseases during self-management, including that on care plans, or unexpected hospitalizations or emergency room visits. Continued feedback from patients would further help improve the utility and quality of the information system.

\section{Conclusion}

This study examined the major factors of care coordination based on the CCM, and further examined the difficulties and practical measures required for introducing the system in South Korea. 
The first consideration in introducing care coordination in South Korea is to form a network among the community and medical service providers. Although there have been numerous attempts to provide comprehensive services for the management of patients with chronic diseases, such efforts have not been successful. In addition to methodological problems and financial difficulties, these attempts could not overcome the problems related to the segmentation of the healthcare delivery system. Financial support is important for securing consent from healthcare providers, but it is more essential to continuously develop the network that is organized in the community, by including a participation mechanism in the decision-making process.

In addition to the lack of experience regarding the management of chronic diseases, there is absolutely no manpower to provide care coordination. Even if a foreign curriculum is introduced in South Korea, it needs to be adapted to the contextual realities of the field. Eventually, the curriculum could be improved as the system is implemented and related experiences are accumulated. However, it is important to note that success of the system is ultimately determined by the quality of the services provided. Therefore, continuous quality control and improvement should be ensured for the services provided in the care coordination system.

It would take time for the care coordination system to settle. All the factors for care coordination described in this study require continuous management and improvement after the actual implementation of the system, including planning for service quality assurance, tracking and managing patients, providing patient support, maintaining consent and agreement on patient referral and transition, and developing and maintaining an electronic information system. Furthermore, most indicators on chronic diseases cannot be improved immediately after the implementation of the system. Therefore, a long-term plan should be established and continuous improvement of the system should be emphasized.

\section{Conflicts of interest}

No potential conflicts of interest relevant to this article was reported.

\section{ORCID}

Jung Jeung Lee, https://orcid.org/0000-0003-0148-7108

Sang Geun Bae, https://orcid.org/0000-0001-7603-7676

\section{References}

1. Oh IH, Yoon SJ, Kim EJ. The burden of disease in Korea. J Korean Med Assoc 2011;54:646-52.

2. Korea Centers for Disease Control \& Prevention. [Korea Health Statistics 2016: Korea National Health and Nutrition Examination Survey (KNHANES VII-1)] [Internet]. Osong: Korea Centers for Disease Control \& Prevention (KCDC); 2017 [cited 2018 Oct 28]. https://knhanes.cdc.go.kr/ knhanes $/$ sub04/sub04_03.do?classType $=7$

3. Cheong W, Yim J, Oh DK, Im JS, Ko KP, Kim YM. Effects of chronic disease management based on clinics for blood pressure or glycemic control in patients with hypertension or type 2 diabetes mellitus. J Agric Med Community Health 2013;38:108-15.

4. Korea Centers for Disease Control \& Prevention. [Hypertension \& diabetes registry project manual] [Internet]. Osong: Korea Centers for Disease Control \& Prevention (KCDC); 2018 [cited 2018 Oct 28]. http://www.cdc.go.kr/ $\mathrm{CDC}$

5. Korea Centers for Disease Control \& Prevention. [The development of model for efficient management of hypertensive patients and diabetics] [Internet]. Osong: Korea Centers for Disease Control \& Prevention (KCDC); 2013 [cited 2018 Oct 28]. http://www. cdc.go.kr/CDC/info/CdcKrInfo0201.jsp?menuIds=HOME001MNU1154-MNU0005-MNU1889\&fid=28\&q type=\&q value $=\&$ cid $=25590 \&$ pageNum $=1$

6. Schraeder C, Shelton P. Comprehensive care coordination for chronically ill adults. Chichester, West Sussex: WileyBlackwell; 2011.

7. Coleman K, Austin BT, Brach C, Wagner EH. Evidence on the Chronic Care Model in the new millennium. Health Aff (Millwood) 2009;28:75-85.

8. Wagner EH, Austin BT, Von Korff M. Organizing care for patients with chronic illness. Milbank Q1996;74:511-44.

9. Wagner EH. The role of patient care teams in chronic disease management. BMJ 2000;320:569-72.

10. Ahmed A. Quality and outcomes of heart failure care in older adults: role of multidisciplinary disease-management programs. J Am Geriatr Soc 2002;50:1590-3.

11. Dorr DA, Wilcox A, Donnelly SM, Burns L, Clayton PD. Impact of generalist care managers on patients with diabetes. Health Serv Res 2005;40:1400-21.

12. Reiss-Brennan B, Briot PC, Savitz LA, Cannon W, Staheli R. Cost and quality impact of Intermountain's mental health integration program. J Healthc Manag 2010;55:97-113.

13. Dickinson KC, Sharma R, Duckart JP, Corson K, Gerrity MS, 
Dobscha SK. VA healthcare costs of a collaborative intervention for chronic pain in primary care. Med Care 2010;48:38-44.

14. Peleg R, Press Y, Asher M, Pugachev T, Glicensztain H, Lederman $\mathrm{M}$, et al. An intervention program to reduce the number of hospitalizations of elderly patients in a primary care clinic. BMC Health Serv Res 2008;8:36.

15. Callahan CM, Boustani MA, Unverzagt FW, Austrom MG, Damush TM, Perkins AJ, et al. Effectiveness of collaborative care for older adults with Alzheimer disease in primary care: a randomized controlled trial. JAMA 2006;295:2148-57.

16. Vickrey BG, Mittman BS, Connor KI, Pearson ML, Della Penna RD, Ganiats TG, et al. The effect of a disease management intervention on quality and outcomes of dementia care: a randomized, controlled trial. Ann Intern Med 2006;145:713-26.

17. Fitzgerald TM, Williams PA, Dodge JA, Quinn M, Heminger CL, Moultrie R, et al. Program implementation approaches to build and sustain health care coordination for type 2 diabetes. Health Promot Pract 2017; 18:306-13.

18. Horner K, Schaefer J, Wagner E. Care coordination: reducing care fragmentation in primary care. In: Phillips KE, Weir V, editor. Safety Net Medical Home Initiative Implementation Guide Series [Internet]. 2nd ed. Seattle, WA: Qualis Health and The MacColl Center for Health Care Innovation at the Group Health Research Institute; 2013 [cited 2018 Oct 28]. http://www.safetynetmedicalhome.org/sites/default/files/ Implementation-Guide-Care-Coordination.pdf

19. Kim J, Hwang YH, Kim JT, Choi NC, Kang SY, Cha JK, et al.
Establishment of government-initiated comprehensive stroke centers for acute ischemic stroke management in South Korea. Stroke 2014;45:2391-6.

20. McDonald K, Schultz E, Albin L, Pineda N, Lonhart J, Sundaram V, et al. Care coordination atlas version 4 (Prepared by Stanford University under subcontract to American Institutes for Research on Contract No. HHSA290-201000005I) [Internet]. Rockville, MD: Agency for Healthcare Research and Quality; 2014 [cited 2018 Oct 28]. https:// fsi-live.s3.us-west-1.amazonaws.com/s3fs-public/atlas june_2014_3.pdf

21. Carrier E, Dowling MK, Pham HH. Care coordination agreements: barriers, facilitators, and lessons learned. Am J Manag Care 2012;18:e398-404.

22. Pham HH, O'Malley AS, Bach PB, Saiontz-Martinez C, Schrag D. Primary care physicians' links to other physicians through Medicare patients: the scope of care coordination. Ann Intern Med 2009; 150:236-42.

23. O'Malley AS, Grossman JM, Cohen GR, Kemper NM, Pham HH. Are electronic medical records helpful for care coordination? Experiences of physician practices. J Gen Intern Med 2010;25:177-85.

24. Berta W, Barnsley J, Bloom J, Cockerill R, Davis D, Jaakkimainen L, et al. Enhancing continuity of information: essential components of consultation reports. Can Fam Physician 2009;55:624-5.

25. Reichman M. Optimizing referrals \& consults with a standardized process. Fam Pract Manag 2007;14:38-42. 